TRANSACTIONS OF THE

AMERICAN MATHEMATICAL SOCIETY

Volume 362, Number 11, November 2010, Pages 5635-5655

S 0002-9947(2010)05275-4

Article electronically published on June 11, 2010

\title{
POLYNOMIALS DEFINING DISTINGUISHED VARIETIES
}

\author{
GREG KNESE
}

\begin{abstract}
Using a sums of squares formula for two-variable polynomials with no zeros on the bidisk, we are able to give a new proof of a representation formula for distinguished varieties. For distinguished varieties with no singularities on the two-torus, we are able to provide extra details about the representation formula and use this to prove a bounded extension theorem.
\end{abstract}

\section{INTRODUCTION}

Let $\mathbb{D}$ be the unit disk, $\mathbb{T}$ be the unit circle, and $\mathbb{E}$ be the set $\mathbb{C} \backslash \overline{\mathbb{D}}$ in $\mathbb{C}$. Let $\mathbb{D}^{2}=\mathbb{D} \times \mathbb{D}$ be the unit bidisk in $\mathbb{C}^{2}$.

Broadly speaking, this paper continues the study of plane algebraic curves (algebraic varieties in $\mathbb{C}^{2}$ ) and their interaction with the two dimensional torus $\mathbb{T}^{2}=$ $\mathbb{T} \times \mathbb{T}$. When viewing curves in this light, one is presented with a number of interesting classes of curves. For example, curves that do not intersect the closed bidisk are described by stable polynomials and are the subject of Geronimo-Woerdeman [9], 10] and Knese [11]. Also, curves $V$ for which $V \cap \mathbb{T}^{2}$ is a determining set for holomorphic functions on $V$ are called toral and are the subject of Agler-McCarthyStankus [5. Both types of curves are intimately related to inner functions and Pick interpolation problems on the bidisk. (Stable polynomials will, in fact, play an important role in this paper later on.)

In this paper we continue the study of a third type of curve, the distinguished varieties.

Definition 1.1. A nonempty set $V$ in $\mathbb{C}^{2}$ is a distinguished variety if there is a polynomial $p \in \mathbb{C}[z, w]$ such that

$$
V=\left\{(z, w) \in \mathbb{D}^{2}: p(z, w)=0\right\}
$$

and such that $V$ exits the bidisk through the distinguished boundary:

$$
\bar{V} \cap \partial\left(\mathbb{D}^{2}\right)=\bar{V} \cap(\partial \mathbb{D})^{2} .
$$

Here we are taking the closure of $V$ in $\overline{\mathbb{D}^{2}}$.

A simple example is the variety $\left\{(z, w) \in \mathbb{D}^{2}: z^{3}=w^{2}\right\}$.

Received by the editors March 25, 2008.

2010 Mathematics Subject Classification. Primary 47A57, 47A13, 14M99, 32A10, 32A60, $14 \mathrm{H} 50$.

Key words and phrases. Distinguished varieties, stable polynomials, bidisk. 
Distinguished varieties were defined in Agler-McCarthy 2] (although they essentially have appeared as far back as W. Rudin [13]). They have also appeared in the paper by P. Vegulla [15] and Agler-McCarthy [3]. Aside from their aesthetic appeal (which we hope to illustrate in this article), here are a few reasons why we think distinguished varieties are interesting.

First, every bounded planar domain with finitely many real analytic boundary curves is biholomorphic to a distinguished variety (this is proven in Vegulla [15, relying on a result of Fedorov [8] on the existence of "unramified separating pairs of inner function"). Hence, studying distinguished varieties provides a unified way of studying function theory on planar domains.

Second, distinguished varieties include many examples of complex spaces with singularities, which have been relatively unstudied in the realm of spaces of analytic functions (and bounded analytic functions more specifically). In many cases, the function theory on a distinguished variety with singularities is isomorphic to function theory on the disk (or some other domain) with some type of constraint imposed (see Agler-McCarthy [4] for more on this). For example, the paper 7 . studies Nevanlinna-Pick interpolation for bounded analytic functions on the disk with the added condition $f^{\prime}(0)=0$. This class of functions is isomorphic to the bounded analytic functions on the distinguished variety $\left\{(z, w) \in \mathbb{D}^{2}: z^{3}=w^{2}\right\}$.

Third, distinguished varieties are important in two-variable matrix theory and in Nevanlinna-Pick interpolation on the bidisk. Indeed, that is the motivation of the original article of Agler-McCarthy [2, and we refer the reader to that paper for more details.

In this paper we give a new proof of a representation formula for distinguished varieties (Theorem 2.1] below) proved in Agler-McCarthy [2. Our new proof is more elementary than the original proof in that it involves studying polynomials that define distinguished varieties directly, as opposed to the approach of the original proof which consisted of constructing certain probability measures on the boundary of the distinguished variety and studying the resulting function theory.

More significantly perhaps, we construct a family of "sums of squares" formulas for polynomials defining distinguished varieties and related polynomials (see Theorems 2.15 and 2.17). These extend known sums of squares formulas for stable polynomials (as in 9] and [11) and polynomials with no zeros on $\mathbb{D}^{2}$ (as in 6] and [11). (See also Theorems 2.11 and 2.13 below.) This approach allows us to get more detailed information about the representation formula for distinguished varieties when the variety in question has no singularities on $\mathbb{T}^{2}$ (see Theorem 2.19).

In turn, this allows us to prove a novel bounded extension theorem (with estimates) for distinguished varieties with no singularities on $\mathbb{T}^{2}$ (see Theorem 2.20). Namely, a polynomial $f$ of two variables on $V$ can be extended to a rational function $F$ whose supremum norm on $\mathbb{D}^{2}$ is bounded by a constant times the supremum norm of $f$ on $V$. The literature is scattered with a number of results of this type; here we mention a few. The paper [14] by Stout gives necessary and sufficient conditions for when such an extension can be performed on discs properly embedded in the polydisc, although due to its more abstract approach does not provide details about constants. The paper Adachi-Andersson-Cho 1 proves extension theorems for analytic subvarieties of analytic polyhedra using integral formulas. We believe our extension theorem, due to its concrete algebraic approach, complements these other results. 


\section{Statements of Results}

We say a two-variable polynomial $p \in \mathbb{C}[z, w]$ has degree $(n, m)$ if it has degree $n$ in $z$ and $m$ in $w$; we say $p$ has degree at most $(n, m)$ if it has degree at most $n$ in $z$ and degree at most $m$ in $w$. Recall that a rational matrix-valued function $\Phi: \mathbb{D} \rightarrow \mathbb{C}^{m \times m}$ on the disk is inner if $\Phi$ is unitary-valued on the unit circle.

The main theorem is the following; see Section 6 for our new proof.

Theorem 2.1 (Agler-McCarthy [2]). Let $V$ be a distinguished variety, defined as the zero set of a polynomial $p \in \mathbb{C}[z, w]$ of minimal degree $(n, m)$. Then, there is an $(m+n) \times(m+n)$ unitary matrix $U$ which we write in block form as

$$
\left.U=\underset{\mathbb{C}^{m}}{\mathbb{C}^{n}} \begin{array}{ll}
\mathbb{C}^{m} & \mathbb{C}^{n} \\
A & B \\
C & D
\end{array}\right)
$$

such that

- D has no unimodular eigenvalues,

- $p(z, w)$ is a constant multiple of

$$
\operatorname{det}\left(\begin{array}{cc}
A-w I_{m} & z B \\
C & z D-I_{n}
\end{array}\right), \text { and }
$$

- defining the following rational matrix-valued inner function:

$$
\Phi(z)=A+z B\left(I_{n}-z D\right)^{-1} C
$$

we have

$$
V=\left\{(z, w) \in \mathbb{D}^{2}: \operatorname{det}\left(w I_{m}-\Phi(z)\right)=0\right\} .
$$

Naturally, the roles of $z$ and $w$ can be reversed above to give similar statements. The original article 22 also proves a converse of the above; namely, if $\Phi$ is a matrixvalued rational inner function on $\mathbb{D}$, then

$$
\left\{(z, w) \in \mathbb{D}^{2}: \operatorname{det}\left(w I_{m}-\Phi(z)\right)\right\}
$$

is a distinguished variety. We are able to provide some additional information about this representation when $V$ has no singularities on $\mathbb{T}^{2}$. This is the content of Theorem 2.19.

We now detail the path to our new proof of Theorem 2.1

Proposition. When a distinguished variety is extended to all of $\mathbb{C}^{2}$ using a defining polynomial $p$ of minimal degree, the resulting variety

$$
V^{\prime}=\left\{(z, w) \in \mathbb{C}^{2}: p(z, w)=0\right\} \subset \mathbb{C}^{2}
$$

satisfies $V^{\prime} \subset \mathbb{D}^{2} \cup \mathbb{T}^{2} \cup \mathbb{E}^{2}$.

This is Proposition 4.1. This gives another way of defining distinguished varieties that is in many ways easier to work with. Namely, instead of the original definition (which looks at subvarieties of $\mathbb{D}^{2}$ ), we can think of distinguished varieties as algebraic subvarieties of $\mathbb{C}^{2}$ satisfying $V \subset \mathbb{D}^{2} \cup \mathbb{T}^{2} \cup \mathbb{E}^{2}$. Once Proposition 4.1 is established we shall think of distinguished varieties in this way and we use the following terminology.

Definition 2.2. We say that a polynomial $p$ defines a distinguished variety if

$$
\left\{(z, w) \in \mathbb{C}^{2}: p(z, w)=0\right\} \subset \mathbb{D}^{2} \cup \mathbb{T}^{2} \cup \mathbb{E}^{2} .
$$


Definition 2.3. Let $q \in \mathbb{C}[z, w]$ be a polynomial of degree at most $(n, m)$. The reflection at the degree $(n, m)$ of $q$ is defined to be the polynomial $\tilde{q}$ given by

$$
\tilde{q}(z, w):=z^{n} w^{m} \overline{q\left(\frac{1}{\bar{z}}, \frac{1}{\bar{w}}\right)} .
$$

If the degree at which reflection is applied is not obvious from the context, we will state the degree of reflection explicitly (see also Remark 2.6).

Notice that $|q(z, w)|=|\tilde{q}(z, w)|$ for all $(z, w) \in \mathbb{T}^{2}$.

Definition 2.4. We say $q \in \mathbb{C}[z, w]$ is essentially $\mathbb{T}^{2}$-symmetric if

$$
q(z, w)=c \tilde{q}(z, w)
$$

for some unimodular constant $c$, and $q$ is $\mathbb{T}^{2}$-symmetric if

$$
q(z, w)=\tilde{q}(z, w) .
$$

Proposition. A polynomial $p$ defining a distinguished variety must be essentially $\mathbb{T}^{2}$-symmetric.

This is Proposition 4.3. An essentially $\mathbb{T}^{2}$-symmetric polynomial can always be multiplied by a unimodular constant to make it $\mathbb{T}^{2}$-symmetric (i.e. if $p=c \tilde{p}$, replace $p$ with $\sqrt{c} p$ for some choice of $\sqrt{c}$ ). Since we are mostly concerned with zero sets, for simplicity we will always make this modification unless otherwise stated.

Because of these facts there is a direct correspondence between polynomials that define distinguished varieties and $\mathbb{T}^{2}$-symmetric polynomials with no zeros on the set $\overline{\mathbb{D}^{2}} \backslash \mathbb{T}^{2}$.

Lemma 2.5. If $p$ is a $\left(\mathbb{T}^{2}\right.$-symmetric) polynomial of degree $(n, m)$ defining a distinguished variety, then

$$
q(z, w):=z^{n} p\left(\frac{1}{z}, w\right)
$$

is a $\mathbb{T}^{2}$-symmetric polynomial of degree $(n, m)$ with no zeros on $\overline{\mathbb{D}^{2}} \backslash \mathbb{T}^{2}$.

See Section 4 for the proof.

It will soon be made apparent why these $\mathbb{T}^{2}$-symmetric polynomials are preferable to polynomials defining distinguished varieties. We shall use the following notation:

$$
\begin{aligned}
q_{z} & =\frac{\partial q}{\partial z} \text { and } q_{w}=\frac{\partial q}{\partial w}, \\
Z_{q} & =\left\{(z, w) \in \mathbb{C}^{2}: q(z, w)=0\right\} .
\end{aligned}
$$

Remark 2.6. When reflecting $q_{z}$ or $q_{w}$ the reflection is assumed to be performed at the degree that would be generically expected. Namely, if $q$ has degree $(n, m)$, then $q_{z}$ is reflected at the degree $(n-1, m)$ :

$$
\widetilde{q_{z}}(z, w)=z^{n-1} w^{m} \overline{q_{z}(1 / \bar{z}, 1 / \bar{w})}
$$

and $q_{w}$ is reflected at the degree $(n, m-1)$. In this case, the reflection of $\widetilde{q}_{z}$ at the degree $(n, m)$ is $z q_{z}(z, w)$.

We mention and prove some identities relating reflection and differentiation in order to demystify the expressions appearing in the theorems to follow. 
Lemma 2.7. Let $q \in \mathbb{C}[z, w]$ be a polynomial of degree at most $(n, m)$. Then,

$$
\begin{aligned}
z \frac{\partial \tilde{q}}{\partial z}(z, w)+\widetilde{q_{z}}(z, w) & =n \tilde{q}(z, w), \\
w \frac{\partial \tilde{q}}{\partial w}(z, w)+\widetilde{q_{w}}(z, w) & =m \tilde{q}(z, w) .
\end{aligned}
$$

In particular, if $q$ is $\mathbb{T}^{2}$-symmetric,

$$
\begin{aligned}
z q_{z}(z, w)+\widetilde{q_{z}}(z, w) & =n q(z, w), \\
w q_{w}(z, w)+\widetilde{q_{w}}(z, w) & =m q(z, w) .
\end{aligned}
$$

Proof. This is a calculus exercise.

Lemma 2.8. If $q \in \mathbb{C}[z, w]$, of degree at most $(n, m)$, is $\mathbb{T}^{2}$-symmetric, then for all $a, b \in \mathbb{R}$,

$$
\begin{aligned}
& (a n+b m)^{2}|q(z, w)|^{2}-2 \operatorname{Re}\left[\left(a z q_{z}(z, w)+b w q_{w}(z, w)\right)(a n+b m) \overline{q(z, w)}\right] \\
& =\left|a \widetilde{q_{z}}(z, w)+b \widetilde{q_{w}}(z, w)\right|^{2}-\left|a z q_{z}(z, w)+b w q_{w}(z, w)\right|^{2} .
\end{aligned}
$$

Proof. By the previous lemma,

$$
a z q_{z}(z, w)+b w q_{w}(z, w)+a \widetilde{q_{z}}(z, w)+b \widetilde{q_{w}}(z, w)=(a n+b m) q(z, w) .
$$

Observe now (omitting the arguments $(z, w)$, i.e. replacing $q(z, w)$ with $q$ )

$$
\begin{aligned}
& (a n+b m)^{2}|q|^{2}-2 \operatorname{Re}\left[\left(a z q_{z}+b w q_{w}\right)(a n+b m) \bar{q}\right] \\
& =\left|a z q_{z}+b w q_{w}+a \widetilde{q_{z}}+b \widetilde{q_{w}}\right|^{2} \\
& \quad-2 \operatorname{Re}\left[\left(a z q_{z}+b w q_{w}\right)\left(\widetilde{a z q_{z}+b w q_{w}+a \widetilde{q_{z}}+b \widetilde{q_{w}}}\right)\right] \\
& =\left|a \widetilde{q_{z}}+b \widetilde{q_{w}}\right|^{2}-\left|a z q_{z}+b w q_{w}\right|^{2} .
\end{aligned}
$$

Theorem 2.9. If $q \in \mathbb{C}[z, w]$ is a $\mathbb{T}^{2}$-symmetric polynomial with no zeros on $\mathbb{D}^{2}$ (resp. $\overline{\mathbb{D}^{2}} \backslash \mathbb{T}^{2}$ ), then $\widetilde{q_{z}}$ and $\widetilde{q_{w}}$ have no zeros on $\mathbb{D}^{2}$ (resp. $\overline{\mathbb{D}^{2}} \backslash \mathbb{T}^{2}$ ). In addition, for all $a, b>0$,

$$
a \widetilde{q_{z}}+b \widetilde{q_{w}}
$$

has no zeros on $\overline{\mathbb{D}^{2}} \backslash\left(Z_{\widetilde{q_{z}}} \cap Z_{\widetilde{q_{w}}}\right)$.

See Section 5 ,

The following corollary (proved in Section (5) is curious because it can be iterated.

Corollary 2.10. If $q \in \mathbb{C}[z, w]$ is an essentially $\mathbb{T}^{2}$-symmetric polynomial of degree $(n, m)$ with no zeros on $\mathbb{D}^{2}$ (or $\left.\overline{\mathbb{D}^{2}} \backslash \mathbb{T}^{2}\right)$, then so is

$$
m n q(z, w)-m z q_{z}(z, w)-n w q_{w}(z, w) .
$$

If $p$ is a polynomial of degree $(n, m)$ defining a distinguished variety, then so is

$$
m z p_{z}(z, w)-n w p_{w}(z, w) .
$$

Theorem 2.9 allows us to use the following sum of squares formula for polynomials with no zeros on the bidisk proved in Cole-Wermer 6. As is articulated in 6, this formula is equivalent to Andô's inequality in operator theory and Agler's Pick interpolation theorem on the bidisk. 
Theorem 2.11 (Cole-Wermer [6]). Let $q \in \mathbb{C}[z, w]$ be a polynomial of degree at most $(n, m)$ with no zeros on the bidisk $\mathbb{D}^{2}$. Then, there exists a vector polynomial

$$
\vec{A}(z, w)=\left(A_{1}(z, w), \ldots, A_{n}(z, w)\right)^{t} \in \mathbb{C}^{n}
$$

of degree at most $(n-1, m)$ (meaning each component is a polynomial of degree at most $(n-1, m))$ and a vector polynomial

$$
\vec{B}(z, w)=\left(B_{1}(z, w), \ldots, B_{m}(z, w)\right)^{t} \in \mathbb{C}^{m}
$$

of degree at most $(n, m-1)$ such that

$$
\begin{aligned}
& q(z, w) \overline{q(Z, W)}-\tilde{q}(z, w) \overline{\tilde{q}(Z, W)} \\
& \quad=(1-z \bar{Z})\langle\vec{A}(z, w), \vec{A}(Z, W)\rangle+(1-w \bar{W})\langle\vec{B}(z, w), \vec{B}(Z, W)\rangle
\end{aligned}
$$

for $(z, w),(Z, W) \in \mathbb{C}^{2}$.

Here $\langle\vec{A}(z, w), \vec{A}(Z, W)\rangle=\sum_{j} A_{j}(z, w) \overline{A_{j}(Z, W)}$ represents the inner product of the two vectors in $\mathbb{C}^{n}, \vec{A}(z, w)$ and $\vec{A}(Z, W)$; we emphasize that this is not any type of Hilbert function space inner product (likewise for $\vec{B}(z, w)$ and $\vec{B}(Z, W)$, elements of $\mathbb{C}^{m}$ ).

More can be said when $q$ in the above theorem has no zeros on the closed bidisk $\overline{\mathbb{D}^{2}}$. Theorem 2.13 below is properly attributed to Geronimo-Woerdeman 9] (see Proposition 2.3.3), although we can more readily explain how this follows from the work in Knese [1] and this is done in the Appendix.

Theorem 2.13 (Geronimo-Woerdeman). If the polynomial $q$ in Theorem 2.11 has no zeros on $\overline{\mathbb{D}^{2}}$, then $\vec{A}$ and $\vec{B}$ may be chosen so that when we write

$$
\begin{aligned}
& \vec{A}(z, w)=A(w)\left(\begin{array}{c}
1 \\
z \\
\vdots \\
z^{n-1}
\end{array}\right), \\
& \vec{B}(z, w)=B(z)\left(\begin{array}{c}
1 \\
w \\
\vdots \\
w^{m-1}
\end{array}\right),
\end{aligned}
$$

where $A(w)$ is an $n \times n$ matrix polynomial in $w$ of degree at most $m$ in each entry and $B(z)$ is an $m \times m$ matrix polynomial in $z$ of degree $n$ in each entry, we have

$$
\begin{aligned}
& A(w) \text { is invertible for all } w \in \overline{\mathbb{D}} \text { and } \\
& z^{n} \overline{B(1 / \bar{z})} \text { is invertible for all } z \in \overline{\mathbb{D}} \text {. }
\end{aligned}
$$

There is an explanation for the apparent asymmetry in this theorem, which would take us too far afield to detail.

Remark 2.14. In a future article, we will extend this theorem to the case where $q$ has no zeros on $\overline{\mathbb{D}^{2}} \backslash \mathbb{T}^{2}$ and finitely many zeros on $\mathbb{T}^{2}$, with the modified conclusion that $A(w)$ as above is invertible for $w \in \overline{\mathbb{D}}$ except possibly at values of $w \in \mathbb{T}$ for which there exists $z \in \mathbb{T}$ such that $q(z, w)=0$ (and similarly for $B(z)$ above).

Here is our sum of squares formula for $\mathbb{T}^{2}$-symmetric polynomials with no zeros on the bidisk. It is proved in Section 5 . 
Theorem 2.15. Suppose $q \in \mathbb{C}[z, w]$ is a $\mathbb{T}^{2}$-symmetric polynomial of degree $(n, m)$ with no zeros on $\mathbb{D}^{2}$ and let $a, b \geq 0$, not both zero. Then, there exists a $\mathbb{C}^{n}$-valued polynomial $\vec{A}$ of degree at most $(\bar{n}-1, m)$ and a $\mathbb{C}^{m}$-valued polynomial $\vec{B}$ of degree at most $(n, m-1)$ such that

$$
\begin{aligned}
(a n+b m)|q(z, w)|^{2} & -2 \operatorname{Re}\left(\left(a z q_{z}(z, w)+b w q_{w}(z, w)\right) \overline{q(z, w)}\right) \\
& =\left(1-|z|^{2}\right)|\vec{A}(z, w)|^{2}+\left(1-|w|^{2}\right)|\vec{B}(z, w)|^{2} .
\end{aligned}
$$

Furthermore, if $q$ is a product of distinct irreducible factors, then $\vec{A}$ and $\vec{B}$ have at most finitely many common zeros on $Z_{q}$.

Here $|\vec{A}(z, w)|$ is the length of the vector $\vec{A}(z, w)$; we emphasize that it is not any type of function space norm.

Remark 2.16. Formulas of the above type can always be polarized to give a formula (as in Theorem 2.11) that is holomorphic in $(z, w)$ and anti-holomorphic in $(Z, W)$. This is the polarization theorem for holomorphic functions which (loosely) says that if $H(z, \bar{z}) \equiv 0$, then $H(z, Z) \equiv 0$ for any holomorphic function $H$ of two variables.

Using the correspondence between distinguished varieties and $\mathbb{T}^{2}$-symmetric polynomials with no zeros on $\overline{\mathbb{D}^{2}} \backslash \mathbb{T}^{2}$, we can prove the following sum of squares formula for polynomials defining distinguished varieties. See Section 5 .

Theorem 2.17. Let $V$ be a distinguished variety given as the zero set of a polynomial $p \in \mathbb{C}[z, w]$ of degree $(n, m)$. Let $a, b \geq 0$, not both zero. Then, there exists a $\mathbb{C}^{n}$-valued vector polynomial $\vec{P}$ of degree at most $(n-1, m)$ and $a \mathbb{C}^{m}$-valued vector polynomial $\vec{Q}$ of degree at most $(n, m-1)$ such that

$$
\begin{aligned}
(b m-a n)|p(z, w)|^{2} & +2 \operatorname{Re}\left[\left(a z p_{z}(z, w)-b w p_{w}(z, w)\right) \overline{p(z, w)}\right] \\
& +\left(1-|z|^{2}\right)|\vec{P}(z, w)|^{2} \\
& =\left(1-|w|^{2}\right)|\vec{Q}(z, w)|^{2} .
\end{aligned}
$$

If $p$ is a product of distinct irreducible factors, then none of the entries of $\vec{P}$ or $\vec{Q}$ can vanish identically on $V$.

Moreover, for $(z, w),(Z, W) \in V$,

$$
(1-z \bar{Z})\langle\vec{P}(z, w), \vec{P}(Z, W)\rangle=(1-w \bar{W})\langle\vec{Q}(z, w), \vec{Q}(Z, W)\rangle .
$$

This last formula (2.18) is all that is needed to prove the representation theorem (and indeed part of the approach in [2] is to prove a similar formula). However, with our approach we are able to "fill out" the formula (2.18) to the rest of $\mathbb{C}^{2}$.

Using Theorem 2.13. we can provide additional information about this representation when $V$ has no singularities on $\mathbb{T}^{2}$. Recall that when a plane curve $V \subset \mathbb{C}^{2}$ is given as the zero set of a polynomial with distinct irreducible factors, it has a singularity at a point in $V$ if and only if both partial derivatives of the defining polynomial vanish at that point.

Theorem 2.19. With all the notation and assumptions as in Theorem 2.1, there is a $\mathbb{C}^{m}$-valued polynomial $\vec{Q}(z, w)$ of degree at most $(n, m-1)$ with at most finitely many zeros on $V$ such that

$$
\Phi(z) \vec{Q}(z, w)=w \vec{Q}(z, w)
$$

for all $(z, w) \in V$. 
In addition, if $V$ has no singularities on $\mathbb{T}^{2}, \vec{Q}$ may be chosen so that when we write

$$
\vec{Q}(z, w)=Q(z)\left(\begin{array}{c}
1 \\
w \\
\vdots \\
w^{m-1}
\end{array}\right),
$$

where $Q(z)$ is an $m \times m$ matrix polynomial of degree at most $n$ in each entry, we have that $Q(z)$ is invertible for all $z \in \overline{\mathbb{D}}$. In particular, $\vec{Q}(z, w)$ has no zeros in $\overline{\mathbb{D}^{2}}$.

As a corollary, we get the following extension theorem.

Theorem 2.20. Let $V$ be a distinguished variety with no singularities on $\mathbb{T}^{2}$ and let $\Phi, Q$, and $\vec{Q}$ be as in Theorem $[2.19$, Then, for any polynomial $f \in \mathbb{C}[z, w]$, the rational function

$$
F(z, w):=(1,0, \ldots, 0) Q(z)^{-1} f\left(z I_{m}, \Phi(z)\right) \vec{Q}(z, w)
$$

is equal to $f$ on $V \cap \overline{\mathbb{D}^{2}}$ and we have the estimates

$$
\begin{aligned}
|F(z, w)| & \leq \| Q(z)^{-1}|||\vec{Q}(z, w)| \sup _{V \cap \mathbb{D}^{2}}|f| \\
& \leq \sqrt{m}|| Q(z)^{-1}\|\| Q(z) \| \sup _{V \cap \mathbb{D}^{2}}|f| \\
& \leq C \sup _{V \cap \mathbb{D}^{2}}|f|
\end{aligned}
$$

for all $(z, w) \in \mathbb{D}^{2}$, where

$$
C=\sqrt{m} \sup _{z \in \mathbb{D}}\left\|Q(z)^{-1}\right\|\|Q(z)\| .
$$

Here we are taking the operator norm of the matrices $Q(z)$ and $Q(z)^{-1}$.

Naturally, the roles of $z$ and $w$ can be reversed in the above theorem, which will sometimes yield a better constant in the extension.

The above theorem clearly produces bounded analytic extensions from $V$ to $\mathbb{D}^{2}$ for functions other than polynomials. Indeed, any function for which we can make sense of $f\left(z I_{m}, \Phi(z)\right)$ on the circle $\mathbb{T}$ will work. We suspect this can be done for all of $H^{\infty}\left(V \cap \mathbb{D}^{2}\right)$, but leave this for future work.

\section{Example of Theorem 2.20}

Let $b(z)$ be a finite Blaschke product and consider the distinguished variety

$$
V=\left\{(z, w) \in \mathbb{D}^{2}: w^{m}-b(z)=0\right\} .
$$

This can be represented as the zero set of

$$
\operatorname{det}\left(w I_{m}-\Phi(z)\right),
$$

where $\Phi$ is the rational matrix-valued inner function

$$
\Phi(z)=\left(\begin{array}{cc}
\mathbf{0} & I_{m-1} \\
b(z) & \mathbf{0}^{t}
\end{array}\right) .
$$


If we set

then

$$
\vec{Q}(z, w)=\left(\begin{array}{c}
1 \\
w \\
\vdots \\
w^{m-1}
\end{array}\right)
$$

$$
\Phi(z) \vec{Q}(z, w)=w \vec{Q}(z, w)
$$

for $(z, w) \in V$. Using our method of estimation from Theorem 2.20 we see that any polynomial $f$ on $V \cap \mathbb{D}^{2}$ can be extended to $\mathbb{D}^{2}$ with its norm increased by at most the factor $\sqrt{m}$. (In this case, $Q(z)$ from Theorem 2.20 is constant and equal to $I_{m}$.)

When $m=2$ and $b(z)=z^{3}$ (i.e. $V=\left\{z^{3}-w^{2}=0\right\}$ ) this improves Theorem 2.9 in Knese [12.

\section{Preliminaries}

As mentioned earlier, the following proposition allows us to think of distinguished varieties in more global terms as subvarieties of $\mathbb{C}^{2}$ satisfying (4.2).

Proposition 4.1. Let $V \subset \mathbb{D}^{2}$ be a distinguished variety defined by a polynomial $p \in \mathbb{C}[z, w]$ of minimal degree $(n, m)$. Then, the extension of $V$ to $\mathbb{C}^{2}$,

$$
V^{\prime}=\left\{(z, w) \in \mathbb{C}^{2}: p(z, w)=0\right\},
$$

satisfies

$$
V^{\prime} \subset \mathbb{D}^{2} \cup \mathbb{T}^{2} \cup \mathbb{E}^{2}
$$

Conversely, if $p$ is a polynomial satisfying

$$
W:=\left\{(z, w) \in \mathbb{C}^{2}: p(z, w)=0\right\} \subset \mathbb{D}^{2} \cup \mathbb{T}^{2} \cup \mathbb{E}^{2},
$$

then $W \cap \mathbb{D}^{2}$ is a distinguished variety.

Proof. The converse statement is clear, so we shall focus on the main claim.

It suffices to prove the proposition for each irreducible component of $V$, so we assume $p$ and $V$ are irreducible. We emphasize that we are starting from the assumption

$$
\bar{V} \cap \partial\left(\mathbb{D}^{2}\right)=\bar{V} \cap \mathbb{T}^{2}
$$

and proving

$$
V^{\prime}=Z_{p} \subset \mathbb{D}^{2} \cup \mathbb{T}^{2} \cup \mathbb{E}^{2}
$$

First, we claim that $p$ can have no zeros on $\mathbb{D} \times \mathbb{T}$. For suppose $p\left(z_{0}, w_{0}\right)=0$ with $\left|z_{0}\right|<1$ and $\left|w_{0}\right|=1$. There is a positive integer $k$ such that for $z$ near $z_{0}$, $p(z, \cdot)$ has a list of zeros $w_{1}(z), w_{2}(z), \ldots, w_{k}(z)$ (listed with possible repetitions) satisfying $w_{j}\left(z_{0}\right)=w_{0}$ and the property that symmetric functions of $w_{1}, \ldots, w_{k}$ are holomorphic near $z_{0}$. (These are all straightforward consequences of the Weierstrass preparation theorem or other theorems about the local behavior of algebraic curves.)

It cannot be the case that all roots $w_{1}(z), w_{2}(z), \ldots, w_{k}(z)$ stay within $\mathbb{T} \cup \mathbb{E}$. One way to see this is to observe that $\pi(z)=\prod_{j=1}^{k} w_{j}(z)$ is holomorphic near $z_{0}$ and $\pi\left(z_{0}\right)=w_{0}^{k}$. If $\pi$ is nonconstant, it must assume a value with modulus less than one, and this implies some sequence of points in $V$ tends to $\mathbb{D} \times \mathbb{T}$, a contradiction. If $\pi$ is constant (and therefore unimodular), either every $w_{j}(z)$ is unimodular-valued (forcing all roots to be constant as a function of $z$, which cannot occur) or some 
$w_{j}(z)$ takes values in $\mathbb{D}$ (which again forces a contradiction) or some $w_{j}(z)$ takes values in $\mathbb{E}$ (and this forces some other $w_{r}(z)$ to assume values in $\left.\mathbb{D}\right)$. Hence, $p$ has no zeros on $\mathbb{D} \times \mathbb{T}$.

The number of zeros of $p(z, \cdot)$ (counting multiplicities) which are contained in $\mathbb{D}$ is constant as a function of $z \in \mathbb{D}$. Indeed,

$$
N:=\int_{\mathbb{T}} \frac{p_{w}(z, w)}{p(z, w)} \frac{d w}{2 \pi i}
$$

is a holomorphic function on the disk which counts the roots of $p(z, \cdot)$ that are contained in $\mathbb{D}$, by the residue theorem. This is valid since $p(z, w)$ is nonzero for $(z, w) \in \mathbb{D} \times \mathbb{T}$. Integer-valued holomorphic functions are constant; hence, $N$ is constant.

For $z \in \mathbb{D}$, let $w_{1}(z), \ldots, w_{N}(z)$ be some listing of the $N$ roots of $p(z, \cdot)$ including multiplicities which are contained in the disk.

From here our strategy will be to show that elementary symmetric functions of the roots of $p(z, \cdot)$ contained in $\mathbb{D}$ are actually rational as a function of $z \in \mathbb{D}$. Recall the elementary symmetric functions are given by

$$
s_{k}(z):=\sum_{1 \leq j_{1}<\cdots<j_{k} \leq N} w_{j_{1}}(z) \cdots w_{j_{k}}(z) .
$$

These are holomorphic on the disk because the functions

$$
f_{k}(z):=\sum_{j=0}^{N}\left(w_{j}(z)\right)^{k}=\int_{\mathbb{T}} \frac{p_{w}(z, w)}{p(z, w)} w^{k} \frac{d w}{2 \pi i}
$$

are holomorphic and the elementary symmetric functions are polynomials in the $f_{k}$.

Since the roots of $p(z, \cdot)$ tend to $\mathbb{T}$ as $z$ tends to $\mathbb{T}$, the Schwarz reflection principle tells us that $s_{N}(z)=w_{1}(z) \cdots w_{N}(z)=\prod_{j=1}^{N} w_{j}(z)$ can be extended meromorphically to the extended complex plane as $1 / \overline{s_{N}(1 / \bar{z})}$ and is therefore rational.

Consider now another family of symmetric polynomials of the roots:

$$
g_{k}(z):=\sum_{j=1}^{N} \prod_{t \neq j} w_{t}(z)^{k} .
$$

Again, $g_{k}$ is holomorphic in the disk. We claim $f_{k}$ extends meromorphically across $\mathbb{T}$ to the function

$$
\hat{f}_{k}(z):=\overline{g_{k}(1 / \bar{z})} / \overline{s_{N}(1 / \bar{z})^{k}}=\sum_{j=1}^{N} \frac{1}{\overline{w_{j}(1 / \bar{z})^{k}}}
$$

defined and meromorphic on $\mathbb{E} \cup\{\infty\}$.

Let $\lambda \in \mathbb{T}$ be a point at which $p(\lambda, \cdot)$ has $m$ distinct roots (only finitely many points fail to satisfy this since $p$ and $p_{w}$ can have no common factor). For $z$ in a small neighborhood of $\lambda, p(z, \cdot)$ has $m$ distinct roots $W_{1}(z), \ldots, W_{m}(z)$ which can be given as holomorphic functions of $z$, by the implicit function theorem. Since $V$ is a distinguished variety, each $W_{j}(z)$ is either always in the disk for $z$ in the disk or always in $\mathbb{E}$ for $z$ in the disk. We may assume the first $N$ of the $W_{j}$ are the roots $w_{1}, \ldots, w_{N}$. Since each $W_{j}(z)$ tends to $\mathbb{T}$ as $z$ tends to $\mathbb{T}$, the Schwarz reflection principle says $W_{j}(z)=1 / \overline{W_{j}(1 / \bar{z})}$ for $z$ in a neighborhood of $\lambda$. Therefore, the function $\sum_{j=0}^{N} W_{j}(z)^{k}$ is holomorphic in a neighborhood of $\lambda$, agrees with $f_{k}(z)$ 
for $z \in \mathbb{D}$, and agrees with $\hat{f}_{k}(z)$ on $\mathbb{E}$. So, $f_{k}$ extends to be meromorphic on the extended complex plane except at possibly finitely many points on $\mathbb{T}$. But, $f_{k}$ is bounded in a neighborhood of the circle and its singularities are therefore removable. Therefore, $f_{k}$ is a rational function. This implies the symmetric functions $s_{k}$ are rational.

Observe that the function

$$
R(z, w)=w^{N}+\sum_{j=0}^{N-1}(-1)^{N-j} s_{N-j}(z) w^{j}
$$

is rational and vanishes on $V$ : it equals

$$
\left(w-w_{1}(z)\right) \cdots\left(w-w_{N}(z)\right)
$$

on the disk and

$$
\left(w-1 / \overline{w_{1}(1 / \bar{z})}\right) \cdots\left(w-1 / \overline{w_{N}(1 / \bar{z})}\right)
$$

on $\mathbb{E}$. Since $p$ is irreducible and $N \leq m$, it must be the case that $N=m$ and the numerator of $R$ is a constant multiple of $p$.

As $R$ was designed to have the property that all roots of $R(z, \cdot)$ are in $\mathbb{D}$ for $z \in \mathbb{D}$ and in $\mathbb{E}$ for $z \in \mathbb{E}$, it follows that

$$
V^{\prime} \subset \mathbb{D}^{2} \cup \mathbb{T}^{2} \cup \mathbb{E}^{2}
$$

Proposition 4.3. If $V$ is a distinguished variety and $V^{\prime}$ is its extension to $\mathbb{C}^{2}$ (as in Proposition 4.1), then $V^{\prime}$ is symmetric with respect to the two-torus: for any $z \neq 0$ and $w \neq 0$,

$$
(z, w) \in V^{\prime} \text { if and only if }\left(\frac{1}{\bar{z}}, \frac{1}{\bar{w}}\right) \in V^{\prime} .
$$

Proof. Assume $V$ is defined as the zero set of a polynomial $p$ of degree $(n, m)$. As before, we may assume $V$ and $p$ are irreducible. It suffices to prove $p$ is essentially $\mathbb{T}^{2}$-symmetric (recall this means $p=c \tilde{p}$ for a unimodular constant $c$ ), since then $p(z, w)=0$ if and only if $p(1 / \bar{z}, 1 / \bar{w})=0$. Write

$$
p(z, w)=\sum_{j=0}^{m} p_{j}(z) w^{j}
$$

for one-variable polynomials $p_{0}, p_{1}, \ldots, p_{m}$ with degrees at most $n$. Then,

$$
\tilde{p}(z, w)=\sum_{j=0}^{m} \tilde{p}_{m-j}(z) w^{j},
$$

where $\tilde{p}_{j}(z)$ is the one-variable reflection of the polynomial $p_{j}$ :

$$
\tilde{p}_{j}(z)=z^{n} \overline{p_{j}\left(\frac{1}{\bar{z}}\right)} \text {. }
$$

For any $z \in \mathbb{T}$, all zeros of $p(z, \cdot)$ are on the circle since $V^{\prime} \subset \mathbb{D}^{2} \cup \mathbb{T}^{2} \cup \mathbb{E}^{2}$. Since

$$
|p(z, w)|=|\tilde{p}(z, w)| \text { for all }(z, w) \in \mathbb{T}^{2}
$$

$p(z, \cdot)$ and $\tilde{p}(z, \cdot)$ have the same zeros when $z \in \mathbb{T}$ (including repeated roots which can be measured by the order of vanishing). 
This implies that for $z \in \mathbb{T}$,

$$
\tilde{p}_{0}(z) p(z, \cdot)=p_{m}(z) \tilde{p}(z, \cdot)
$$

since the leading coefficients of these two polynomials with the same zeros are equal; i.e. for $(z, w) \in \mathbb{T} \times \mathbb{C}$,

$$
\tilde{p}_{0}(z) p(z, w)=p_{m}(z) \tilde{p}(z, w) .
$$

This can only happen if (4.4) holds for all $(z, w) \in \mathbb{C}^{2}$, since $\mathbb{T}^{2}$ is a set of uniqueness for polynomials.

By irreducibility of $p$ we see that $p=c \tilde{p}$ for some constant $c$. Since $|p|=|\tilde{p}|$ on $\mathbb{T}^{2}$, the constant $c$ must be unimodular. This proves $p$ is essentially symmetric.

Proof of Lemma 2.5. Let $p$ be a polynomial of degree $(n, m)$ defining the distinguished variety $V$ in the sense of Definition 2.2. Then,

$$
q(z, w):=z^{n} p\left(\frac{1}{z}, w\right)=w^{m} \overline{p\left(\bar{z}, \frac{1}{\bar{w}}\right)}
$$

defines a polynomial of degree strictly equal to $(n, m)$. (The middle expression has degree $m$ in $w$ and the expression on the right has degree $n$ in $z$.)

Also, by equation (4.5), since $p$ has no zeros on the set

$$
(\mathbb{E} \times \mathbb{D}) \cup(\mathbb{D} \times \mathbb{E}) \cup(\mathbb{T} \times \mathbb{D}) \cup(\mathbb{D} \times \mathbb{T}),
$$

it follows that $q$ has no zeros on $\overline{\mathbb{D}^{2}} \backslash \mathbb{T}^{2}$ except possibly at $(0,0)$. Since zeros of two-variable polynomials are never isolated, we may conclude $q$ has no zeros on $\overline{\mathbb{D}^{2}} \backslash \mathbb{T}^{2}$.

Finally, since $p$ is essentially $\mathbb{T}^{2}$-symmetric, it is clear that $q$ is too.

\section{SUMS OF SQUARES FORMUlAS}

In this section we prove Theorems 2.9, 2.15 and 2.17 We will identify $V$ with its extension $V^{\prime}$ to $\mathbb{C}^{2}$ from the previous section via a defining polynomial of minimal degree.

Proof of Theorem 2.9 , Let $q$ be a $\mathbb{T}^{2}$-symmetric polynomial with no zeros on $\mathbb{D}^{2}$ (or $\overline{\mathbb{D}^{2}} \backslash \mathbb{T}^{2}$ ). For each $t \in(0,1]$ define

$$
q_{t}(z, w):=q(t z, w)
$$

(not to be confused with the partial derivative notation). Then, $q_{t}$ has no zeros on $\mathbb{D}^{2}$ and therefore

$$
\left|q_{t}(z, w)\right|^{2}-\left|\widetilde{q}_{t}(z, w)\right|^{2} \geq 0 \text { on } \overline{\mathbb{D}^{2}} .
$$

Since $q$ is $\mathbb{T}^{2}$-symmetric, it can be shown that

$$
\widetilde{\left(q_{t}\right)}(z, w)=t^{n} q(z / t, w) .
$$

Considering the following expression for $(z, w) \in \overline{\mathbb{D}^{2}}$,

$$
\frac{|q(t z, w)|^{2}-\left|t^{n} q(z / t, w)\right|^{2}}{1-t^{2}} \geq 0,
$$

and taking the limit as $t \nearrow 1$ yields

$$
n|q(z, w)|^{2}-2 \operatorname{Re}\left(z q_{z}(z, w) \overline{q(z, w)}\right) \geq 0 \text { on } \overline{\mathbb{D}^{2}} .
$$


By the Lemma 2.8 with $a=1, b=0$,

$$
\begin{aligned}
& n^{2}|q(z, w)|^{2}-2 \operatorname{Re}\left(z q_{z}(z, w) \overline{n q(z, w)}\right) \\
& =\left|\widetilde{q}_{z}(z, w)\right|^{2}-\left|z q_{z}(z, w)\right|^{2} \geq 0 \text { on } \overline{\mathbb{D}^{2}} .
\end{aligned}
$$

Therefore, any zero of $\widetilde{q_{z}}$ on $\overline{\mathbb{D}^{2}}$ must also be a zero of $z q_{z}$, and by the identity $n q=\widetilde{q_{z}}+z q_{z}$ (from Lemma 2.7), any zero of $\widetilde{q_{z}}$ on $\overline{\mathbb{D}^{2}}$ is a zero of $q$. So, if $q$ has no zeros on $\mathbb{D}^{2}$ (resp. $\overline{\mathbb{D}^{2}} \backslash \mathbb{T}^{2}$ ), then $\widetilde{q_{z}}$ has no zeros on $\mathbb{D}^{2}$ (resp. $\overline{\mathbb{D}^{2}} \backslash \mathbb{T}^{2}$ ). Similar statements hold for $\widetilde{q_{w}}$.

By Lemma 2.8 (used three times), when $a, b \geq 0$ and $(z, w) \in \overline{\mathbb{D}^{2}}$ we have

$$
\begin{aligned}
& \left|a \widetilde{q_{z}}+b \widetilde{q_{w}}\right|^{2}-\left|a z q+b w q_{w}\right|^{2} \\
& =(a n+b m)\left[(a n+b m)|q|^{2}-2 \operatorname{Re}\left[\left(a z q_{z}+b w q_{w}\right) \bar{q}\right]\right] \\
& =(a n+b m)\left[(a / n)\left(n^{2}|q|^{2}-2 \operatorname{Re}\left(z q_{z} n \bar{q}\right)\right)+(b / m)\left(m^{2}|q|^{2}-2 \operatorname{Re}\left(w q_{w} m \bar{q}\right)\right)\right] \\
& =(a n+b m)\left[(a / n)\left(\left|\widetilde{q_{z}}\right|^{2}-\left|z q_{z}\right|^{2}\right)+(b / m)\left(\left|\widetilde{q_{w}}\right|^{2}-\left|w q_{w}\right|^{2}\right)\right] \\
& \geq(a n+b m)\left[(a / n)\left(1-|z|^{2}\right)\left|\widetilde{q_{z}}\right|^{2}+(b / m)\left(1-|w|^{2}\right)\left|\widetilde{q_{w}}\right|^{2}\right]
\end{aligned}
$$

where the inequality follows from

$$
\begin{aligned}
& \left|\widetilde{q_{z}}\right|^{2}-\left|z q_{z}\right|^{2}=\left|\widetilde{q_{z}}\right|^{2}\left(1-|z|^{2}\right)+|z|^{2}\left(\left|\widetilde{q_{z}}\right|^{2}-\left|q_{z}\right|^{2}\right) \\
& \geq\left(1-|z|^{2}\right)\left|\widetilde{q_{z}}\right|^{2} .
\end{aligned}
$$

(Note: for brevity we are omitting the argument $(z, w)$ in front of all of the polynomials above.)

Since $\widetilde{q_{z}}$ and $\widetilde{q_{w}}$ have no zeros on $\mathbb{D}^{2}$, it now follows that for $a, b>0, a \widetilde{q_{z}}+b \widetilde{q_{w}}$ has no zeros on $\mathbb{D}^{2}$. If $a \widetilde{q_{z}}+b \widetilde{q_{w}}$ has a zero on $\overline{\mathbb{D}^{2}} \backslash \mathbb{D}^{2}$, then the left side of (5.1) vanishes to at least order two. This implies $\left(1-|z|^{2}\right)\left|\widetilde{q_{z}}\right|^{2}$ and $\left(1-|w|^{2}\right)\left|\widetilde{q_{w}}\right|^{2}$ both vanish to order at least two. Since $\left(1-|z|^{2}\right)$ and $\left(1-|w|^{2}\right)$ vanish to at most order 1 at a point in $\overline{\mathbb{D}^{2}} \backslash \mathbb{D}^{2}$, it follows that both $\widetilde{q_{z}}$ and $\widetilde{q_{w}}$ vanish at a zero of $a \widetilde{q_{z}}+b \widetilde{q_{w}}$. Therefore, $a \widetilde{q_{z}}+b \widetilde{q_{w}}$ has no zeros on $\overline{\mathbb{D}^{2}} \backslash\left(Z_{\widetilde{q_{z}}} \cap Z_{\widetilde{q_{w}}}\right)$ when $a, b>0$.

Proof of Corollary 2.10. There is no harm in assuming $q$ is $\mathbb{T}^{2}$-symmetric. Setting $a=m$ and $b=n$ in (5.1) yields

$$
\begin{aligned}
& 2 \operatorname{Re}\left(\left(n m q(z, w)-m z q_{z}(z, w)-n w q_{w}(z, w) \overline{q(z, w)}\right)\right. \\
& \geq(m / n)\left(1-|z|^{2}\right)\left|\widetilde{q_{z}}(z, w)\right|^{2}+(n / m)\left(1-|w|^{2}\right)\left|\widetilde{q_{w}}(z, w)\right|^{2} \geq 0
\end{aligned}
$$

on $\mathbb{D}^{2}$. Since $\widetilde{q_{z}}$ and $\widetilde{q_{w}}$ have no zeros on $\mathbb{D}^{2}$ (resp. $\overline{\mathbb{D}^{2}} \backslash \mathbb{T}^{2}$ ) when $q$ has no zeros on $\mathbb{D}^{2}$ (resp. $\overline{\mathbb{D}^{2}} \backslash \mathbb{T}^{2}$ ), it follows that

$$
n m q(z, w)-m z q_{z}(z, w)-n w q_{w}(z, w)
$$

has no zeros on $\mathbb{D}^{2}$ (resp. $\overline{\mathbb{D}^{2}} \backslash \mathbb{T}^{2}$ ) when $q$ has no zeros on $\mathbb{D}^{2}$ (resp. $\overline{\mathbb{D}^{2}} \backslash \mathbb{T}^{2}$ ).

By Lemma 2.7 the reflection of this polynomial at the degree $(n, m)$ is equal to

$$
-n m q(z, w)+m z q_{z}(z, w)+n w q_{w}(z, w),
$$

and therefore

$$
n m q(z, w)-m z q_{z}(z, w)-n w q_{w}(z, w)
$$

is essentially $\mathbb{T}^{2}$-symmetric.

The statement that $m z p_{z}-n w p_{w}$ defines a distinguished variety when $p$ defines a distinguished variety follows by applying the previous arguments to $q(z, w)=$ 
$z^{n} p(1 / z, w)$ and then converting back to expressions involving $p$ and $p$ 's partial derivatives.

Proof of Theorem 2.15. Apply Theorem 2.11 to $a \widetilde{q_{z}}+b \widetilde{q_{w}}$ and use the formula in Lemma 2.8 to prove the existence of $\vec{A}$ and $\vec{B}$ satisfying

$$
\begin{aligned}
(a n+b m)|q(z, w)|^{2} & -2 \operatorname{Re}\left(\left(a z q_{z}(z, w)+b w q_{w}(z, w)\right) \overline{q(z, w)}\right) \\
& =\left(1-|z|^{2}\right)|\vec{A}(z, w)|^{2}+\left(1-|w|^{2}\right)|\vec{B}(z, w)|^{2} .
\end{aligned}
$$

This can be polarized into the equation

$$
\begin{aligned}
& (a n+b m) q(z, w) \overline{q(Z, W)} \\
& -\left(a z q_{z}(z, w)+b w q_{w}(z, w) \overline{q(Z, W)}\right. \\
& -q(z, w) \overline{a Z q_{z}(Z, W)+b W q_{w}(Z, W)} \\
& =(1-z \bar{Z})\langle\vec{A}(z, w), \vec{A}(Z, W)\rangle+(1-w \bar{W})\langle\vec{B}(z, w), \vec{B}(Z, W)\rangle .
\end{aligned}
$$

Suppose now that $q$ is a product of distinct irreducible factors. Suppose $\vec{A}$ and $\vec{B}$ have infinitely many zeros on $Z_{q}$. This means they must have a factor in common with $q$. Specifically, $q=f g$ for some relatively prime polynomials $f$ and $g$, and $f$ divides $\vec{A}$ and $\vec{B}$. Substituting $(Z, W)=(0,0)$ into (5.2) and rearranging produces

$$
\begin{aligned}
(a n+b m) q(z, w) \overline{q(0,0)} & -\langle\vec{A}(z, w), \vec{A}(0,0)\rangle-\langle\vec{B}(z, w), \vec{B}(0,0)\rangle \\
& =\left(a z q_{z}(z, w)+b w q_{w}(z, w) \overline{q(0,0)} .\right.
\end{aligned}
$$

The polynomial $f$ divides the left hand side and hence divides $a z q_{z}+b w q_{w}$ (note $q(0,0) \neq 0)$. Using $q=f g$ we have

$$
\begin{aligned}
a z q_{z}(z, w)+b w q_{w}(z, w)= & \left(a z f_{z}(z, w)+b w f_{w}(z, w)\right) g(z, w) \\
& +\left(a z g_{z}(z, w)+b w g_{w}(z, w)\right) f(z, w)
\end{aligned}
$$

and therefore $f$ divides $a z f_{z}+b w f_{w}$ since $f$ and $g$ are relatively prime. Since the degree of $a z f_{z}+b w f_{w}$ is less than or equal to the degree of $f$ (in each variable separately) and since $f(0,0) \neq 0$, this implies $a z f_{z}+b w f_{w} \equiv 0$. As all factors of $q$ are essentially $\mathbb{T}^{2}$-symmetric polynomials with no zeros on $\mathbb{D}^{2}$ (and can be made $\mathbb{T}^{2}$-symmetric), applying the formula in Lemma 2.8 to $f$ we arrive at the contradictory conclusion that $f \equiv 0$.

Thus, $\vec{A}$ and $\vec{B}$ can only have finitely many zeros on $Z_{q}$ when $q$ is a product of distinct irreducible factors.

Proof of Theorem 2.17. By Lemma 2.5. Theorem 2.15 can be applied to $q$ given by

$$
q(z, w):=z^{n} p(1 / z, w) .
$$

Before doing so we list some simple formulas relating partial derivatives of $q$ and $p$ :

$$
\begin{aligned}
z q_{z}(z, w) & =n z^{n} p(1 / z, w)-z^{n-1} p_{z}(1 / z, w), \\
w q_{w}(z, w) & =z^{n} p_{w}(1 / z, w)
\end{aligned}
$$

and therefore

$$
\begin{aligned}
a z q_{z}(z, w)+b w q_{w}(z, w) & =a n z^{n} p(1 / z, w)-\left(a z^{n-1} p_{z}(1 / z, w)-b w z^{n} p_{w}(1 / z, w)\right) \\
& =a z^{n} \widetilde{p}_{z}(1 / z, w)+b w z^{n} p_{w}(1 / z, w) .
\end{aligned}
$$


By Theorem 2.15] using equation (5.3), there exists a $\mathbb{C}^{n}$-valued polynomial $\vec{A}(z, w)$ of degree at most $(n-1, m)$ and a $\mathbb{C}^{m}$-valued polynomial $\vec{B}(z, w)$ of degree at most $(n, m-1)$ with finitely many common zeros on $Z_{q}$ such that

$$
\begin{aligned}
& (a n+b m)\left|z^{n} p(1 / z, w)\right|^{2} \\
& -2 \operatorname{Re}\left(\left[a n z^{n} p(1 / z, w)-\left(a z^{n-1} p_{z}(1 / z, w)-b w z^{n} p_{w}(1 / z, w)\right)\right] \overline{z^{n} p(1 / z, w)}\right) \\
& =\left(1-|z|^{2}\right)|\vec{A}(z, w)|^{2}+\left(1-|w|^{2}\right)|\vec{B}(z, w)|^{2} .
\end{aligned}
$$

Replacing $z$ with $1 / z$ and multiplying through by $\left|z^{n}\right|^{2}$ yields

$$
\begin{aligned}
& (a n+b m)|p(z, w)|^{2}-2 \operatorname{Re}\left(\left(a n p(z, w)-\left(a z p_{z}(z, w)-b w p_{w}(z, w)\right)\right) \overline{p(z, w)}\right) \\
& =(b m-a n)|p(z, w)|^{2}+2 \operatorname{Re}\left(\left(a z p_{z}(z, w)-b w p_{w}(z, w)\right) \overline{p(z, w)}\right) \\
& =\left(|z|^{2}-1\right)\left|z^{n-1} \vec{A}(1 / z, w)\right|^{2}+\left(1-|w|^{2}\right)\left|z^{n} \vec{B}(1 / z, w)\right|^{2} .
\end{aligned}
$$

Defining $\vec{P}(z, w)=z^{n-1} \vec{A}(1 / z, w)$ and $\vec{Q}(z, w)=z^{n} \vec{B}(1 / z, w)$, we get

$$
\begin{aligned}
& (b m-a n)|p(z, w)|^{2}+2 \operatorname{Re}\left(\left(a z p_{z}(z, w)-b w p_{w}(z, w)\right) \overline{p(z, w)}\right) \\
& +\left(1-|z|^{2}\right)|\vec{P}(z, w)|^{2} \\
& =\left(1-|w|^{2}\right)|\vec{Q}(z, w)|^{2} .
\end{aligned}
$$

By the polarization theorem for holomorphic functions, we get the formula:

$$
\begin{aligned}
(b m-a n) p(z, w) \overline{p(Z, W)} & +\left(a z p_{z}(z, w)-b w p_{w}(z, w)\right) \overline{p(Z, W)} \\
& +(1-z \bar{Z})\langle\vec{P}(z, w), \vec{P}(Z, W)\rangle \\
& =p(z, w)\left(b \overline{W p_{w}(Z, W)}-a \overline{Z p_{z}(Z, W)}\right) \\
& +(1-w \bar{W})\langle\vec{Q}(z, w), \vec{Q}(Z, W)\rangle .
\end{aligned}
$$

For $(z, w),(Z, W) \in V$, the above reduces to

$$
(1-z \bar{Z})\langle\vec{P}(z, w), \vec{P}(Z, W)\rangle=(1-w \bar{W})\langle\vec{Q}(z, w), \vec{Q}(Z, W)\rangle .
$$

The fact that none of the components of $\vec{P}$ or $\vec{Q}$ is identically zero when the degree $(n, m)$ is minimal will follow from our proof of Theorem 2.1. For the moment, we at least know that $\vec{P}$ and $\vec{Q}$ have finitely many common zeros on $V$. (There is no circular reasoning going on here as will be made apparent at the end of the proof of Theorem 2.1.)

\section{REPRESENTATION OF Distinguished VARIETIES}

Proof of Theorem 2.1: We continue directly from the end of the proof of Theorem 2.17. Equation (5.5) can be rearranged into

$$
\begin{aligned}
& \langle\vec{P}(z, w), \vec{P}(Z, W)\rangle+w \bar{W}\langle\vec{Q}(z, w), \vec{Q}(Z, W)\rangle \\
& =z \bar{Z}\langle\vec{P}(z, w), \vec{P}(Z, W)\rangle+\langle\vec{Q}(z, w), \vec{Q}(Z, W)\rangle
\end{aligned}
$$

and from here much of the proof follows a standard "lurking isometry"/systems theory argument similar to that found in [2].

The map sending

$$
\left(\begin{array}{c}
\vec{Q}(z, w) \\
z \vec{P}(z, w)
\end{array}\right) \mapsto\left(\begin{array}{c}
w \vec{Q}(z, w) \\
\vec{P}(z, w)
\end{array}\right)
$$


for each $(z, w) \in V$ defines a unitary on the span of elements of $\mathbb{C}^{m+n}$ of the form on the left to the span of the elements of $\mathbb{C}^{m+n}$ of the form on the right which we may extend to a unitary matrix $U: \mathbb{C}^{m} \oplus \mathbb{C}^{n} \rightarrow \mathbb{C}^{m} \oplus \mathbb{C}^{n}$. Let us write $U$ in block form:

$$
U=\left(\begin{array}{ll}
A & B \\
C & D
\end{array}\right)
$$

Then,

$$
\begin{aligned}
& A \vec{Q}(z, w)+z B \vec{P}(z, w)=w \vec{Q}(z, w), \\
& C \vec{Q}(z, w)+z D \vec{P}(z, w)=\vec{P}(z, w)
\end{aligned}
$$

for all $(z, w) \in V$.

This implies

$$
\left(\begin{array}{l}
\mathbf{0} \\
\mathbf{0}
\end{array}\right)=\left(\begin{array}{cc}
A-w I_{m} & z B \\
C & z D-I_{n}
\end{array}\right)\left(\begin{array}{l}
\vec{Q}(z, w) \\
\vec{P}(z, w)
\end{array}\right)
$$

for all $(z, w) \in V$. By Theorem 2.15 and by construction, $\vec{P}$ and $\vec{Q}$ have at most finitely many common zeros on $V$. Therefore,

$$
\operatorname{det}\left(\begin{array}{cc}
A-w I_{m} & z B \\
C & z D-I_{n}
\end{array}\right)=0
$$

for all but finitely many points on $V$ and hence all points on $V$. Since this is a polynomial of degree at most $(n, m)$, it must equal a constant multiple of $p$, by minimality of $p$. This proves the first representation formula in Theorem 2.1.

Equation (6.2) implies

$$
\left(I_{n}-z D\right)^{-1} C \vec{Q}(z, w)=\vec{P}(z, w),
$$

and coupled with equation (6.1) we have

$$
\left\{A+z B\left(I_{n}-z D\right)^{-1} C-w I_{m}\right\} \vec{Q}(z, w)=0 \text { for }(z, w) \in V .
$$

Define $\Phi(z):=A+z B\left(I_{n}-z D\right)^{-1} C$. A well-known calculation proves $\Phi(z)$ is a rational inner function. Indeed, the fact that $U$ is a unitary and that

$$
U\left(\begin{array}{c}
I_{m} \\
z\left(I_{n}-z D\right)^{-1} C
\end{array}\right)=\left(\begin{array}{c}
\Phi(z) \\
\left(I_{n}-z D\right)^{-1} C
\end{array}\right)
$$

implies

$I_{m}+|z|^{2} C^{*}\left(I_{n}-\bar{z} D^{*}\right)^{-1}\left(I_{n}-z D\right)^{-1} C=\Phi(z)^{*} \Phi(z)+C^{*}\left(I_{n}-\bar{z} D^{*}\right)^{-1}\left(I_{n}-z D\right)^{-1} C$.

Rearranging gives

$$
I_{m}-\Phi(z)^{*} \Phi(z)=\left(1-|z|^{2}\right) C^{*}\left(I_{n}-\bar{z} D^{*}\right)^{-1}\left(I_{n}-z D\right)^{-1} C .
$$

Claim 6.4. $D$ has no unimodular eigenvalues.

Proof. Suppose there is a nonzero vector $\vec{v} \in \mathbb{C}^{n}$ such that $D \vec{v}=\lambda \vec{v}$ for some $\lambda \in \mathbb{T}$. Then,

$$
U\left(\begin{array}{l}
\mathbf{0} \\
\vec{v}
\end{array}\right)=\left(\begin{array}{c}
B \vec{v} \\
\lambda \vec{v}
\end{array}\right)
$$


and the unitarity of $U$ implies $B \vec{v}=0$. Hence, $\left(\begin{array}{l}\mathbf{0} \\ \vec{v}\end{array}\right)$ is an eigenvector for $U$ and after a unitary change of coordinates (which will not affect the $\mathbb{C}^{m}$ portion of $\mathbb{C}^{m} \oplus \mathbb{C}^{n}$ ), $U$ can be put into the form

$$
\left(\begin{array}{lll}
A & B^{\prime} & \mathbf{0} \\
C^{\prime} & D^{\prime} & \mathbf{0} \\
\mathbf{0}^{t} & \mathbf{0}^{t} & \lambda
\end{array}\right)
$$

This implies $p$ is a constant multiple of

$$
(\lambda z-1) \operatorname{det}\left(\begin{array}{cc}
A-w I_{m} & z B^{\prime} \\
C^{\prime} & z D^{\prime}-I_{n-1}
\end{array}\right)
$$

which contradicts the fact that $p$ defines a distinguished variety.

By the claim, it now follows that $\Phi(z)^{*} \Phi(z)=I_{m}$ for $z \in \mathbb{T}$. In words, $\Phi$ is unitary-valued on the circle.

On $V \cap \mathbb{D}^{2}$, the zeros of $\vec{Q}$ coincide with the zeros of $\vec{P}$ by equation (5.5). Hence, we see that $\vec{Q}$ has at most finitely many zeros on $V$. So, by (6.3),

$$
\operatorname{det}\left(w I_{m}-\Phi(z)\right)=0
$$

for all $(z, w) \in V$. The rational function

$$
r(z, w)=\operatorname{det}\left(w I_{m}-\Phi(z)\right)
$$

has numerator with degree at most $m$ in $w$ and it vanishes on $V$, and this implies that it must be a constant multiple of $p$. As was already mentioned above,

$$
\Phi(z) \vec{Q}(z, w)=w \vec{Q}(z, w) \text { for }(z, w) \in V,
$$

and this completes the proof of Theorem 2.1.

If any of the components of $\vec{P}$ or $\vec{Q}$ had been identically zero, this same proof could have yielded a polynomial of strictly lower degree than $p$ (in either $z$ or $w)$ which vanished on $V$. This cannot happen since $p$ is by definition minimal. Therefore, we have also completed the proof Theorem 2.17.

Proof of Theorem 2.19. The existence of $\vec{Q}$ as stated in the theorem follows from the previous proof.

If $V=Z_{p}$ has no singularities on $\mathbb{T}^{2}$, then $p_{z}$ and $p_{w}$ have no common zeros on $V \cap \mathbb{T}^{2}$. Using the formulas $n p=z p_{z}+\widetilde{p_{z}}$ and $m p=w p_{w}+\widetilde{p_{w}}$, all zeros of $p_{z}$ and $p_{w}$ on $\mathbb{T}^{2}$ must occur on $V$. Hence, $p_{z}$ and $p_{w}$ have no common zeros on all of $\mathbb{T}^{2}$. Reverting to $\mathbb{T}^{2}$-symmetric polynomials with no zeros on $\overline{\mathbb{D}^{2}} \backslash \mathbb{T}^{2}$ :

$$
q(z, w):=z^{n} p(1 / z, w),
$$

we see that $q_{z}$ and $q_{w}$ have no common zeros on $\mathbb{T}^{2}$. By Theorem 2.9 , $\widetilde{q_{z}}$ and $\widetilde{q_{w}}$ each have no zeros on $\overline{\mathbb{D}^{2}} \backslash \mathbb{T}^{2}$. So, again by Theorem 2.9 , $\widetilde{q_{z}}+\widetilde{q_{w}}$ has no zeros on $\overline{\mathbb{D}^{2}} \backslash\left(Z_{\widetilde{q_{z}}} \cap Z_{\widetilde{q_{w}}}\right)=\overline{\mathbb{D}^{2}}$.

Theorem 2.13 can be applied to $\widetilde{q_{z}}+\widetilde{q_{w}}$, and the proof of Theorem 2.15 can be repeated with the additional knowledge that $\vec{B}(z, w)$, when written in matrix form as

$$
\vec{B}(z, w)=B(z)\left(\begin{array}{c}
1 \\
w \\
\vdots \\
w^{m-1}
\end{array}\right),
$$


has the property that $z^{n} \overline{B(1 / \bar{z})}$ is invertible for $z \in \overline{\mathbb{D}}$. Repeating the proof of Theorem 2.17, we see that

$$
\vec{Q}(z, w)=z^{n} w^{m-1} \overline{\vec{B}(1 / \bar{z}, 1 / \bar{w})}=z^{n} \overline{B(1 / \bar{z})}\left(\begin{array}{c}
w^{m-1} \\
w^{m-2} \\
\vdots \\
1
\end{array}\right),
$$

and therefore the matrix $Q(z)$ satisfying

$$
\vec{Q}(z, w)=Q(z)\left(\begin{array}{c}
1 \\
w \\
\vdots \\
w^{m-1}
\end{array}\right)
$$

is equal to $z^{n} \overline{B(1 / \bar{z})}$ with its columns in reverse order. Thus, $Q(z)$ is invertible for $z \in \overline{\mathbb{D}}$.

Proof of Theorem $\left[2.20\right.$. If $f \in \mathbb{C}[z, w]$, then for any vectors $\overrightarrow{v_{1}}, \overrightarrow{v_{2}} \in \mathbb{C}^{m}$ we have that for each $z \in \mathbb{D}$,

$$
\begin{aligned}
\left|\left\langle f\left(z I_{m}, \Phi(z)\right) \overrightarrow{v_{1}}, \overrightarrow{v_{2}}\right\rangle\right| & \leq \sup _{\lambda \in \mathbb{T}}\left|\left\langle f\left(\lambda I_{m}, \Phi(\lambda)\right) \overrightarrow{v_{1}}, \overrightarrow{v_{2}}\right\rangle\right| \\
& \leq \sup _{V \cap \mathbb{D}^{2}}|f|\left|\overrightarrow{v_{1}}\right|\left|\overrightarrow{v_{2}}\right|
\end{aligned}
$$

by the maximum modulus principle and since $f\left(\lambda I_{m}, \Phi(\lambda)\right)$ is normal with eigenvalues given by the values of $f$ on $V$ when $\lambda \in \mathbb{T}$ (recall that $\Phi$ is unitary on the circle). Therefore, in the operator norm,

$$
\left\|f\left(z I_{m}, \Phi(z)\right)\right\| \leq \sup _{V \cap \mathbb{D}^{2}}|f| .
$$

Now, we examine

$$
F(z, w):=(1,0, \ldots, 0) Q(z)^{-1} f\left(z I_{m}, \Phi(z)\right) \vec{Q}(z, w) .
$$

For $(z, w) \in V \cap \mathbb{D}^{2}, f\left(z I_{m}, \Phi(z)\right) \vec{Q}(z, w)=f(z, w) \vec{Q}(z, w)$, and therefore when $(z, w) \in V \cap \mathbb{D}^{2}$,

$$
F(z, w)=f(z, w)(1,0, \ldots, 0) Q(z)^{-1} Q(z)\left(\begin{array}{c}
1 \\
w \\
\vdots \\
w^{m-1}
\end{array}\right)=f(z, w) ;
$$

i.e. $F$ is an extension of $f$.

The estimates on $F$ are now straightforward:

$$
\begin{aligned}
|F(z, w)| & \leq\left\|Q(z)^{-1}\right\|\left\|f\left(z I_{m}, \Phi(z)\right)\right\||\vec{Q}(z, w)| \\
& \leq\left\|Q(z)^{-1}\right\||\vec{Q}(z, w)| \sup _{V \cap \mathbb{D}^{2}}|f| \\
& \leq \sqrt{m}\left\|Q(z)^{-1}\right\|\|Q(z)\| \sup _{V \cap \mathbb{D}^{2}}|f|
\end{aligned}
$$


for all $(z, w) \in \mathbb{D}^{2}$ since

$$
|\vec{Q}(z, w)|=\left|Q(z)\left(\begin{array}{c}
1 \\
w \\
\vdots \\
w^{m-1}
\end{array}\right)\right| \leq \sqrt{m}|| Q(z)||
$$

for $|w| \leq 1$.

\section{Appendix}

In this appendix we explain how to obtain Theorem 2.13 from the results in Knese [11. Let $q$ be a polynomial of degree at most $(n, m)$ with no zeros on $\overline{\mathbb{D}^{2}}$.

Define a probability measure $\rho$ on $\mathbb{T}^{2}$ by

$$
d \rho=\frac{c^{2}}{|q(z, w)|^{2}} \frac{d z}{2 \pi i} \frac{d w}{2 \pi i},
$$

where $c$ is chosen to make $\rho$ a bona fide probability measure. Let $\langle\cdot, \cdot\rangle_{\rho}$ denote the standard inner product on $L^{2}(\rho)$. Consider the following $n$-dimensional subspace of 2-variable polynomials defined using the given inner product:

$$
\begin{aligned}
S_{1} & =\{\text { polynomials of degree at most }(n-1, m)\} \\
& \ominus_{\rho}\{w p(z, w): p \text { is a polynomial of degree at most }(n-1, m-1)\},
\end{aligned}
$$

where we have written $\ominus_{\rho}$ to emphasize that this is an orthogonal complement performed using $\langle,\rangle_{\rho}$.

Claim 7.1. No nonzero element of $S_{1}$ is divisible by a polynomial of the form $L(z, w)=w-w_{0}$, where $w_{0} \in \overline{\mathbb{D}}$.

Proof. Suppose $L$ divides a nonzero $p \in S_{1}$; i.e. $p(z, w)=\left(w-w_{0}\right) r(z, w)$ for some polynomial $r$ of degree at most $(n-1, m-1)$. Then,

$$
\begin{aligned}
\left|w_{0}\right|^{2}\|r\|_{L^{2}(\rho)}^{2} & =\left\|w_{0} r\right\|_{L^{2}(\rho)}^{2}=\|p-w r\|_{L^{2}(\rho)}^{2} \\
& =\|p\|_{L^{2}(\rho)}^{2}+\|r\|_{L^{2}(\rho)}^{2}
\end{aligned}
$$

since $p$ is orthogonal to $w r$ and since multiplication by $w$ is an isometry on $L^{2}(\rho)$. (We are slightly abusing notation here and confounding $w$ with the function $(z, w)$ $\mapsto w$.) Therefore, $\|p\|_{L^{2}(\rho)}^{2}=\left(\left|w_{0}\right|^{2}-1\right)\|r\|_{L^{2}(\rho)}^{2}$, which can only be positive when $\left|w_{0}\right|>1$.

Let $K S_{1}((z, w),(Z, W))$ be the reproducing kernel for $S_{1}$ using the given inner product $\langle,\rangle_{\rho}$ (for details on reproducing kernels in this setting, see [11]). For any orthonormal basis $\left\{E_{1}, \ldots, E_{n}\right\}$ of $S_{1}$ it is a fact that

$$
K S_{1}((z, w),(Z, W))=\sum_{j=1}^{n} E_{j}(z, w) \overline{E_{j}(Z, W)}
$$

or in vector polynomial notation with $\vec{E}(z, w)=\left(E_{1}(z, w), \ldots, E_{n}(z, w)\right)^{t}$ we have

$$
K S_{1}((z, w),(Z, W))=\langle\vec{E}(z, w), \vec{E}(Z, W)\rangle .
$$


Writing $\vec{E}(z, w)$ in the matrix form

$$
\vec{E}(z, w)=E(w)\left(\begin{array}{c}
1 \\
z \\
\vdots \\
z^{n-1}
\end{array}\right),
$$

we make the following claim.

Claim 7.3. The $n \times n$ matrix $E(w)$ is invertible for all $w \in \overline{\mathbb{D}}$.

Proof. Suppose $E(w)$ is singular for some $w_{0} \in \overline{\mathbb{D}}$. Then, there is a nonzero vector $\vec{v} \in \mathbb{C}^{n}$ such that $\vec{v}^{t} E\left(w_{0}\right)=\mathbf{0}^{t}$. This implies $\vec{v}^{t} \vec{E}(z, w) \in S_{1}$ vanishes on the set $w=w_{0}$. By the previous claim this can only occur if $\vec{v}^{t} \vec{E}$ is identically zero, which cannot happen since $\left\{E_{1}, \ldots, E_{n}\right\}$ is a basis for $S_{1}$ and $\vec{v}$ is nonzero.

Consider a second subspace

$$
\begin{aligned}
\widetilde{S_{2}} & :=\{\text { polynomials of degree }(n, m-1)\} \\
& \ominus_{\rho}\{\text { polynomials of degree }(n-1, m-1)\} .
\end{aligned}
$$

Using arguments similar to the above it can be shown that the reproducing kernel $K \widetilde{S_{2}}$ can be written as

$$
K \widetilde{S_{2}}((z, w),(Z, W))=\langle\vec{F}(z, w), \vec{F}(Z, W)\rangle,
$$

where $\vec{F}$ is a $\mathbb{C}^{m}$-valued polynomial which when written in matrix form

$$
\vec{F}(z, w)=F(z)\left(\begin{array}{c}
1 \\
w \\
\vdots \\
w^{m-1}
\end{array}\right)
$$

has the property that $z^{n} \overline{F(1 / \bar{z})}$ is invertible for all $z \in \overline{\mathbb{D}}$.

Finally we can give the connection to Theorem 2.13. Theorems 4.5 and 5.1 in Knese [1] say

$$
\frac{|q(z, w)|^{2}}{c^{2}}-\frac{|\tilde{q}(z, w)|^{2}}{c^{2}}=\left(1-|z|^{2}\right)|\vec{E}(z, w)|^{2}+\left(1-|w|^{2}\right)|\vec{F}(z, w)|^{2},
$$

which easily implies Theorem 2.13 by the above discussion.

\section{REFERENCES}

1. Kenzō Adachi, Mats Andersson, and Hong Rae Cho. $L^{p}$ and $H^{p}$ extensions of holomorphic functions from subvarieties of analytic polyhedra. Pacific J. Math., 189(2):201-210, 1999. MR:1696119 (2000c:32036)

2. Jim Agler and John E. McCarthy. Distinguished varieties. Acta Math., 194(2):133-153, 2005. MR2231339(2007c:47006)

3. Jim Agler and John E. McCarthy. Parametrizing distinguished varieties. In Recent advances in operator-related function theory, volume 393 of Contemp. Math., pages 29-34. Amer. Math. Soc., Providence, RI, 2006. MR2198369 (2007i:32012)

4. Jim Agler and John E. McCarthy. Hyperbolic algebraic and analytic curves. Indiana Univ. Math. J., 56(6):2899-2933, 2007. MR2375707 (2009a:32039)

5. Jim Agler, John E. McCarthy, and Mark Stankus. Toral algebraic sets and function theory on polydisks. J. Geom. Anal., 16(4):551-562, 2006. MR 2271943(2007j:32002)

6. Brian J. Cole and John Wermer. Ando's theorem and sums of squares. Indiana Univ. Math. J., 48(3):767-791, 1999. MR.1736979(2000m:47014) 
7. Kenneth R. Davidson, Vern I. Paulsen, Mrinal Raghupathi, and Dinesh Singh. A Constrained Nevanlinna-Pick Interpolation Problem. Indiana Univ. Math. J. 58(2):709-732, 2009. MR 2514385

8. S. I. Fedorov. Harmonic analysis in a multiply connected domain. I. Mat. Sb., 181(6):833-864, 1990. MR $1072300(92 \mathrm{~m}: 30068 \mathrm{a})$

9. Jeffrey S. Geronimo and Hugo J. Woerdeman. Positive extensions, Fejér-Riesz factorization and autoregressive filters in two variables. Ann. of Math. (2), 160(3):839-906, 2004. MR 2144970 (2006b:42036)

10. Jeffrey S. Geronimo and Hugo J. Woerdeman. Two-variable polynomials: intersecting zeros and stability. IEEE Trans. Circuits Syst. I Regul. Pap., 53(5):1130-1139, 2006. MR 2235187 (2007c:93050)

11. Greg Knese. Bernstein-Szegő measures on the two dimensional torus. Indiana Univ. Math. J. 57(3):1353-1376, 2008. MR2429095 (2009h:46054)

12. Greg Knese. Function theory on the Neil parabola. Michigan Math. J., 55(1):139-154, 2007. MR2320176 (2008b:32004)

13. Walter Rudin. Pairs of inner functions on finite Riemann surfaces. Trans. Amer. Math. Soc., 140:423-434, 1969. MR0241629 (39:2968)

14. Edgar Lee Stout. Bounded extensions. The case of discs in polydiscs. J. Analyse Math., 28:239254, 1975. MR0590066 (58:28662)

15. Prasad Vegulla. Geometry of distinguished varieties. to appear in Michigan Math. J.

Department of Mathematics, University of California, Irvine, Irvine, California 92614-3875

E-mail address: gknese@uci.edu

URL: http://www.math.uci.edu/ gknese

Current address: Department of Mathematics, University of Alabama, Box 870350, Tuscaloosa, Alabama 35487-0350

E-mail address: gknese@bama.ua.edu 